\title{
Total Synthesis of ( \pm )-Phomactin B2 via an Intramolecular Cyclohexadienone Annulation of a Chromium Carbene Complex
}

\author{
Jie Huang, Chunrui Wu, and William D. Wulff \\ Department of Chemistry, Michigan State University, East Lansing, MI 48824.
}

\begin{abstract}
A total synthesis of $( \pm)$-phomactin B2 is described which has as its key step the intramolecular cyclohexadienone annulation of a Fischer carbene complex. The requisite carbene complex was prepared from geraniol in 11 steps and $12 \%$ overall yield. The key cyclohexadienone annulation produced both rings of the [9.3.1] pentadecane ring system of phomactin B2 in a single step in 60\% yield and as a 4:1 mixture of diastereomers. The major diastereomer was taken on to the natural product in a series of steps that begins with a Peterson olefination. Initially, the Peterson olefination failed but x-ray analysis of two intermediates in the diastereomeric series revealed that approach to the hindered carbonyl was blocked by a TIPS protecting group. Replacement of the TIPS group with a MOM group led to a facile Peterson olefination. Another notable steps in the synthesis included a stereoselective methylation of a cyclohexenone and hydroxyl directed epoxidation of an alkene.
\end{abstract}

Phomactins are novel platelet activating factor (PAF) antagonists isolated from the culture of marine fungus Phoma sp. ${ }^{1}$ Members of the phomactin family share a unique [9.3.1] pentadecane ring system and their biosynthetic pathway shares a branchpoint with the taxane family of natural products. ${ }^{1,2}$ Substantial synthetic efforts have been described in the literature towards the synthesis of several phomactins, ${ }^{3}$ but of the eleven known members of the phomactin family, total syntheses have only been reported for phomactins A, D and G. ${ }^{4}$ The nexus to all of the published retrosynthetic plans, realized or no, is the construction of the sixmembered ring prior to the 12 membered ring. We report here a different approach to the phomactins that involves the simultaneous assembly of both the six and 12-membered rings via an intramolecular cyclohexadienone annulation of a chromium carbene complex and which is rendered to practice in a total synthesis of $( \pm)$-phomactin B2.

The retrosynthetic plan for the synthesis of phomactin B2 presented in Scheme 1 targets the cyclohexadienone $\mathbf{2}$ as a key intermediate. Access to $\mathbf{2}$ was envisioned to be possible from the thermolysis of carbene complex $\mathbf{3}$ which should initiate loss of a carbon monoxide ligand and subsequently an intramolecular reaction of the carbene complex with the alkyne function to generate a cyclohexadienone. The carbene complex $\mathbf{3}$ was in turn envisioned to be preparable from geraniol.

The cyclohexadienone annulation of chiral propargyl ethers is known to generate significant 1,4-asymmetric induction in favor of the diastereomer $7 .{ }^{5}$ We have recently reported the first examples of an intramolecular cyclohexadienone annulation with the finding that moderate to good yields of 9 can be obtained when the number of methylenes in the tether of carbene complex 8 is 8 or greater. ${ }^{3}$ Since there are 9 carbons in the macrocyclic bridge in the phomactins, the propitious model studies with $\mathbf{8}$ set the stage for the enactment of the strategy in Scheme 1.

E-mail: wulff@ chemistry.msu.edu. 
The first stage of the synthesis involves the preparation of the carbene complex 3 which begins with geraniol and its conversion to the known bromide 10 in 4 steps in $49 \%$ overall yield. ${ }^{6}$ Three carbons were then introduced via coupling with 1-trimethylsilyl propargyl lithium which after deprotection gives $\mathbf{1 1}$ in 78\% yield. The E-vinyl iodide in intermediate $\mathbf{1 2}$ was then installed by a Negishi carbometalation. ${ }^{7}$ The control of this stereochemistry is important since the model studies with carbene complex $\mathbf{8}$ reveal that the E-isomers are much more efficient than their Z-couterparts. ${ }^{3}$ The terminal alkyne unit is readily installed via oxidation of the allylic alcohol 12 and then reaction with ethynyl Grignard. The resulting alcohol is protected in two forms such that carbene complexes $\mathbf{3 a}$ and $\mathbf{3 b}$ can both be evaluated. The carbene complexes are prepared by the Fischer method ${ }^{8}$ but the generation of a dianion from $\mathbf{1 4}$ can be problematic. To prevent metal/halogen exchange prior to depronation of the alkyne, phenyllithium is used as base prior to the addition of $n$-BuLi. Carbene complex $3 \mathbf{a}$ could be obtained in reproducible yields of 50\% over a range of scales if the chromium carbonyl and alkynyl iodide $\mathbf{1 4}$ where mixed prior to the addition of phenyllithium. If the dianion is generated and then reacted with $\mathrm{Cr}(\mathrm{CO})_{6}$ the yields of $\mathbf{3 a}$ are unpredictable and range from $0-50 \%$.

The intramolecular cyclohexadienone annulation of complex 3a gave a mixture of the diastereomers 2a and 15a in a ratio that was temperature dependent. The highest diastereomeric ratio of $4: 1$ was observed at $60^{\circ} \mathrm{C}$ and while this is on the low side observed for intermolecular reactions, ${ }^{5}$ it is slightly higher than we have observed for intramolecular reactions in model systems. ${ }^{3}$ The relative configuration was determined by an X-ray diffraction study on the more crystalline isomer 15a.
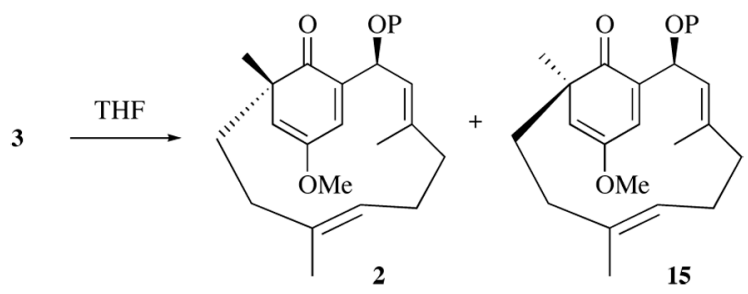

The completion of the synthesis of phomactin B2 from $\mathbf{2 a}$ begins with the installation of the exo-cyclic double-bond. It was anticipated that the quaternary carbon adjacent to the carbonyl group in 2a may substantially reduce its reactivity and accordingly, it was found not to react with trimethylsilyl-methyllithium after $48 \mathrm{~h}$ at room temperature. Heating leads to decomposition. Surprisingly, the diastereomer 15a reacts in 15 minutes under the same conditions to give the 1,2-adduct in high yield. A possible explanation comes from the X-ray structures of 15a and alcohol $\mathbf{2 c}$ ( $2 \mathbf{a}$ is not crystalline). The conformation about the $\mathrm{C}-\mathrm{C}$ bond connecting the propargyl ether carbon with the 6-membered ring has the methine hydrogen of the propargyl carbon anti to the carbonyl in 15a and syn to the carbonyl in $\mathbf{2 c}$ (and thus presumably in 2a). Thus, the difference in reactivity between $\mathbf{2 a}$ and $\mathbf{1 5} \mathbf{a}$ may be to the degree to which the TIPS group interferes with the approach of the nucleophile along the Burgi Dunitz trajectory. If this is true, then replacing the TIPS group in 2a with a MOM group should lead to a chelation assisted approach of $\mathrm{TMSCH}_{2} \mathrm{Li}$ to the carbonyl. Indeed, $\mathbf{2} \mathbf{b}$ readily yielded to the Peterson protocol giving $\mathbf{1 6}$ after hydrolysis of the enol ether. The alternative to changing the protecting group in $\mathbf{2} \mathbf{a}$ is the direct generation of $\mathbf{2} \mathbf{b}$ from the carbene complex $\mathbf{3 b}$ but this is not viable since there is a complete loss in stereoselectivity and a dramatic drop in yield (Table 1). The methylation of the enolate of $\mathbf{1 6}$ gives a single diastereomer with the methyl group anti to the macrocyclic tether as expected. The MOM protecting group proved to be a two-edged sword since it interfered with the subsequent 1,2-reduction of the ketone giving substantial contamination with a 1,6-reduction product. The remedy was the reinstallation of the TIPS group which then allowed clean reduction to $\mathbf{1 7}$ as an inseparable 2:1 mixture of isomers. The hydroxyl group in $\mathbf{1 7}$ was protected as an acetate in a effort to electronically quell 
competing epoxidation of the wrong allylic alcohol. The acetates could be separated and were epoxidized ${ }^{9}$ independently. The epoxide from 19 was oxidized to a ketone and hydrolyzed to give Phomactin B2. The same transformations on the epoxide 21 gives keto alcohol 22. The fact that $\mathbf{2 2}$ can be converted to Phomactin B2 by a Mitsunobu inversion reveals that the face selectivity of the epoxidation is independent of the acetate configuration and it increases the convergency of the synthesis since both $\mathbf{1 8}$ and $\mathbf{1 9}$ can be taken to product.

We are currently attempting to define an asymmetric approach based on this strategy and to apply the general strategy described here to the synthesis of other members of the phomactin family.

\section{Supplementary Material}

Refer to Web version on PubMed Central for supplementary material.

\section{Acknowledgment}

This work was supported by a grant from the NIH (GM 33589).

\section{REFERENCES}

1. For a review of the phomactins, see: Goldring WPD, Pattenden G. Acc. Chem. Res 2006;39:354-361. [PubMed: 16700534]

2. (a) Tokiwano T, Fukushi E, Endo T, Oikawa H. Chem. Comm 2004:1324-1325. [PubMed: 15154058] (b) Tokiwano T, Endo T, Tsukagoshi T, Goto H, Fukushi E, Oikawa H. Org. Biomol. Chem 2005:2713-2722. [PubMed: 16032349] (c) Chow SY, Williams HJ, Pennington JD, Nanda S, Reibenspies JH, Scott AI. Tetrahedron 2007;63:6204-6209.

3. For leading citations, see: Huang J, Wang H, Wu C, Wulff WD. Org. Lett 2007;9:2799-2802. [PubMed: 17580880]

4. For the total syntheses of phomactins, see: Phomactin D; (a) Miyaoka H, Saka Y, Miura S, Yamada Y. Tetrahedron Lett 1996;37:7107-7110. Phomactin A; (b) Goldring WPD, Pattenden G. Chem. Comm 2002:1736-1737. [PubMed: 12196974] (c) Mohr PJ, Halcomb RL. J. Am. Chem. Soc 2003;125:1712-1713. [PubMed: 12580592] (d) Diaper CM, Goldring WPD, Pattenden G. Org. Biomol. Chem 2003:3949-3956. [PubMed: 14664384] Phomactin G; (e) Goldring WPD, Pattenden G. Org. Biomol. Chem 2004:466-473. [PubMed: 14770224]

5. Hsung RP, Quinn JF, Weisenberg BA, Wulff WD, Yap GPA, Rheingold AL. Chem. Commun 1997:615-616.

6. Armstrong R, Weiler L. Can. J. Chem 1983;61:2530-2539.

7. Negishi E, Horn DE, Yoshida T. J. Am. Chem. Soc 1985;107:6639-6647.

8. Aumann R, Fischer EO. Chem. Ber 1968;101:954-962.

9. Hirao T. Chem. Rev 1997;97:2707-2724. [PubMed: 11851478] 


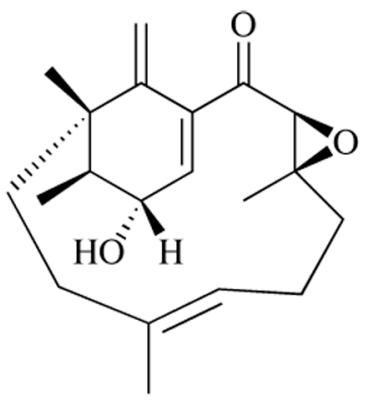

1 Phomactin B2

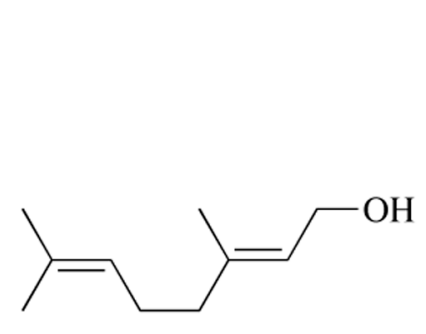

4 Geraniol

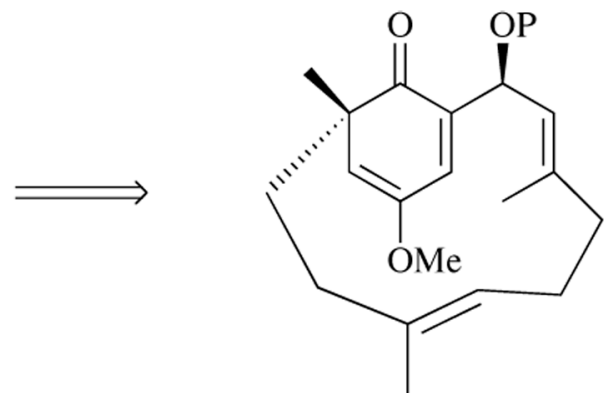

2

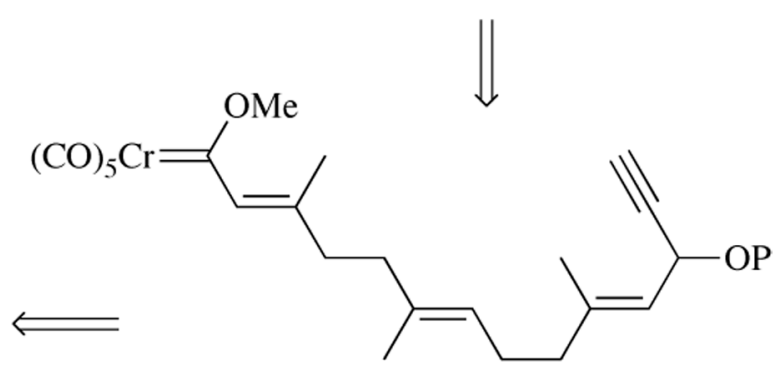

3

Scheme 1. 


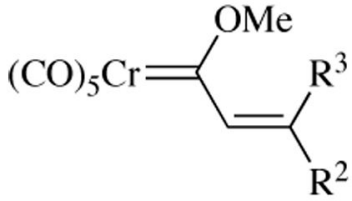

5

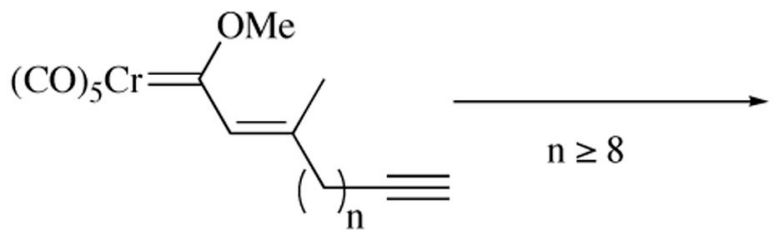

8
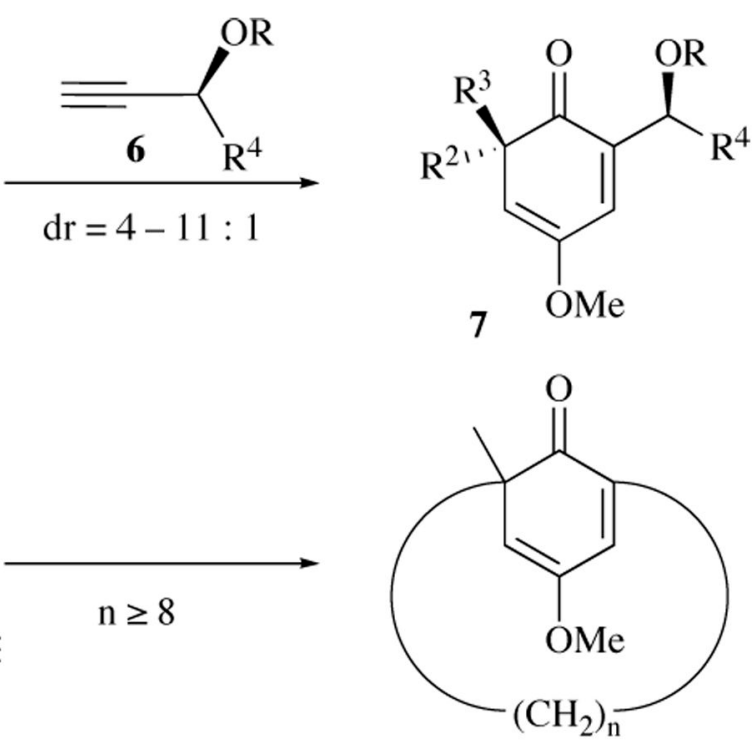

9

Scheme 2. 


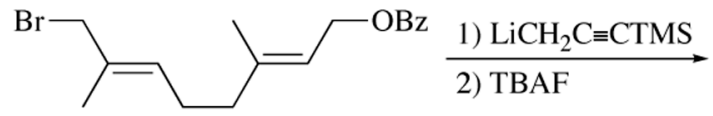

10

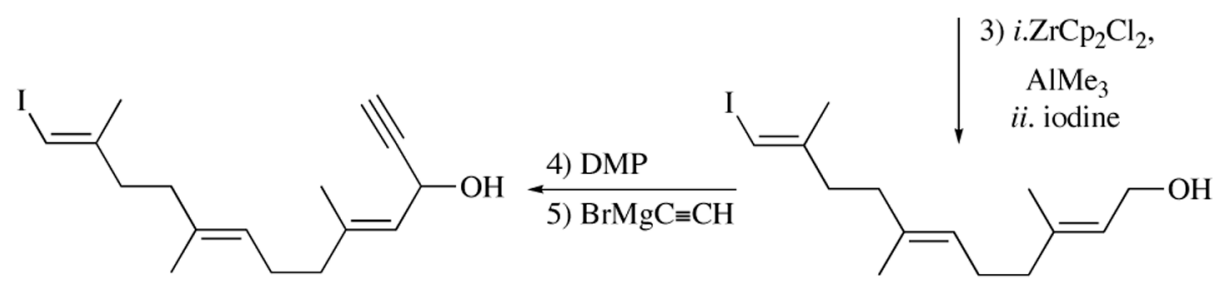

$1391 \%$

6) TIPSCI, DMAP

or

$\mathrm{MOMCl}, i-\mathrm{Pr}_{2} \mathrm{EtN}$

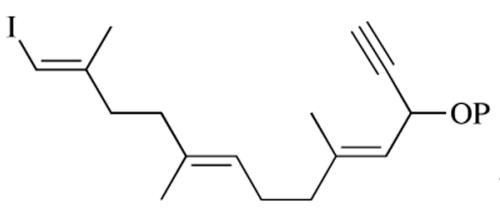

14a $100 \% \mathrm{P}=$ TIPS

14b $80 \% \quad \mathrm{P}=\mathrm{MOM}$
7) $i . \mathrm{Cr}(\mathrm{CO})_{6}, \mathrm{THF},-78^{\circ} \mathrm{C}$

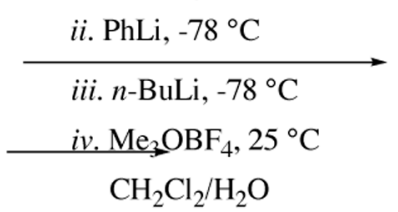

$\mathrm{CH}_{2} \mathrm{Cl}_{2} / \mathrm{H}_{2} \mathrm{O}$ 3a $51 \% \quad \mathrm{P}=$ TIPS 3b $43 \% \quad \mathrm{P}=\mathrm{MOM}$

Scheme 3. 


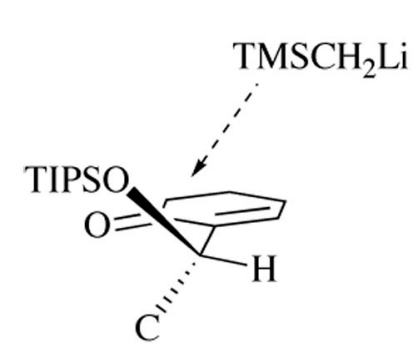

conformation in $\mathbf{1 5 a}$

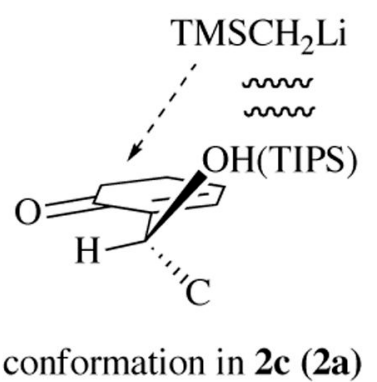

conformation in $\mathbf{2 c}(\mathbf{2 a})$

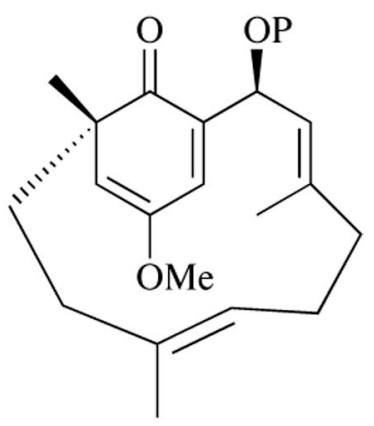

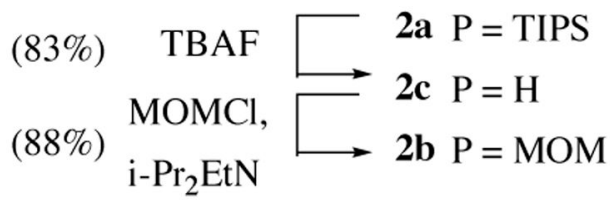

Scheme 4. 


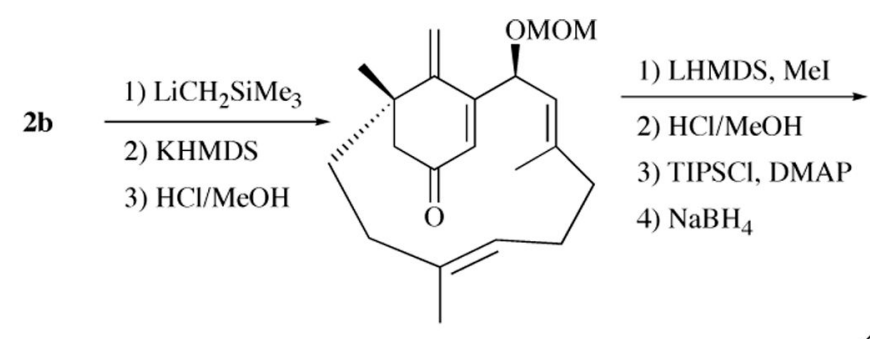

$1674 \%$

1) $\mathrm{VO}(\mathrm{acac})_{2}$<smiles>C=C1C([C@H](O)C2OC2(C)CCC=C(C)CO)=C[C@H](O)[C@@H]2CCC1C2</smiles>

$2188 \%$

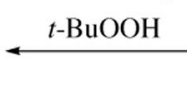<smiles>C=C1C2=C[C@H](CC2)[C@@H](O)C=C1[C@H](O)/C=C(\C)CCC=C(C)C</smiles>

18

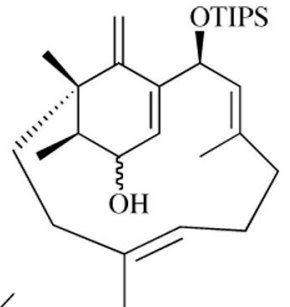

$17(81 \%)$

1) $\mathrm{Ac}_{2} \mathrm{O}$, pyr

2) $\mathrm{TBAF}$

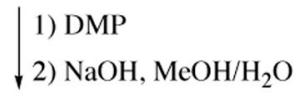

$(70 \%)$

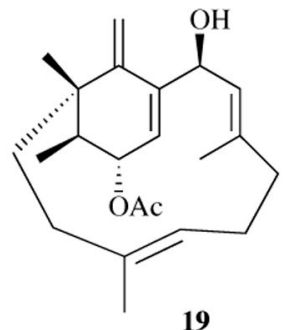

1) $\mathrm{VO}(\mathrm{acac})_{2}$
$t-\mathrm{BuOOH}$

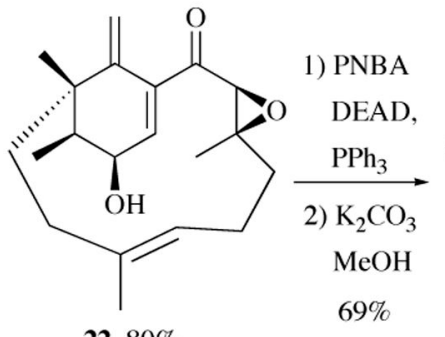

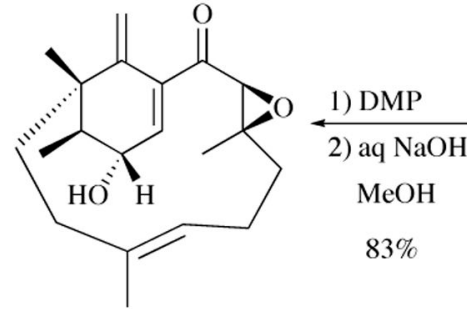

1 Phomactin B2

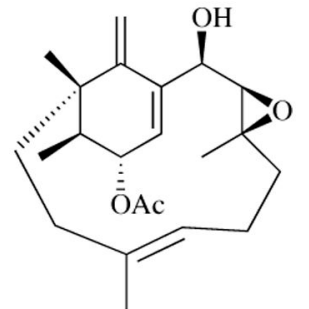

20 93\%

Scheme 5. 


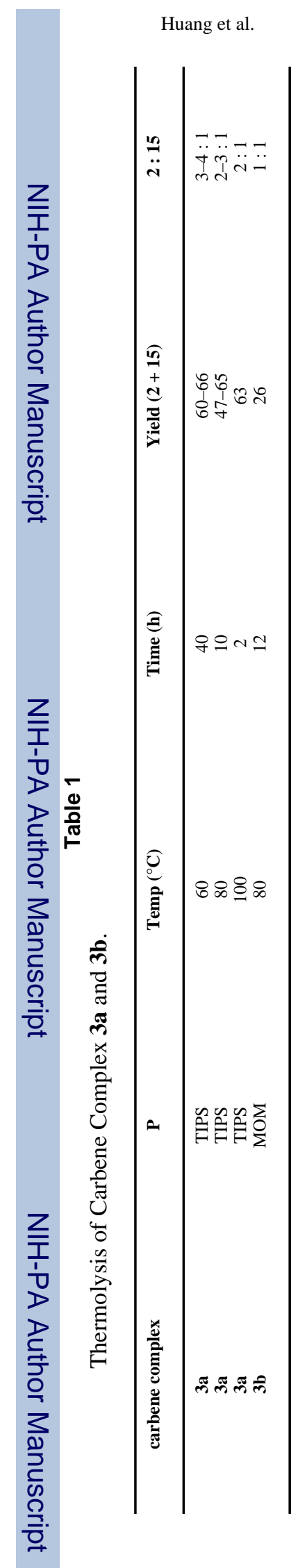

Page 9 